RESEARCH REPORT

\title{
EFFICACY OF TENS WITH CONVENTIONAL OCCUPATIONAL THERAPY IN IMPROVING HAND FUNCTION OF STROKE SURVIVORS
}

\begin{abstract}
BACKGROUND AND AIM

Stroke is currently the second leading cause of death and third main cause of disability throughout the world. Around $75 \%$ of stroke survivors suffered from upper limb dysfunction with limited functional activities whereas distal parts of upper extremity such as hand and forearm are mostly affected by stroke. Therefore, this study aims to determine the benefits of TENS along with conventional Occupational therapy in the rehabilitation of stroke patients to ascertain the optimal recovery of hand function.
\end{abstract}

\section{METHODOLOGY}

A Single-blind Randomized Controlled Trial was conducted on 76 individuals with acute phase or one-year history post-stroke. Group-A $(n=38)$ received Low Frequency Transcutaneous Electrical Nerve Stimulation on elbow and wrist extensors $(3 \mathrm{~Hz}, 400 \mu \mathrm{s})$ along with conventional Occupational therapywhile Group-B ( $n=38)$ received conventional Occupational therapy only. Both the groups received intervention for 5 days/week of duration 45 minutes till 3 weeks.

\section{RESULTS}

Group-A showed significant improvement in means scores of FMA-UE and ARAT $(p<0.05)$ in comparison to Group-B which showed moderate improvements in sensorimotor and activity capacity of hand function.

\section{CONCLUSION}

Conventional therapy along with TENS significantly improved the hand function in acute stroke patients as compared to the conventional therapy.

\section{KEYWORDS}

Electrical stimulation, Transcutaneous Electric Nerve Stimulation, Conventional therapy, Stroke, Upper extremity, Activity limitations.

\author{
Faiza Tahir \\ Occupational Therapist \\ Seyada Rehabilitation Center \\ alitahir436@yahoo.com

\section{Quratulain Khan} \\ Clinical Occupational Therapist \\ Institute of Physical Medicine and Rehabili- \\ tation (IPM\&R) \\ DUHS \\ quratulainkhan05@gmail.com
}

[Tahir F, Khan QA. Efficacy of TENS with conventional Occupational Therapy in Improving Hand Function of Stroke Survivors. Pak.j.rehabil. 2019;8(1):25-30] 


\section{INTRODUCTION}

Globally 12.6 million people are permanently disabled due to stroke'. Stroke is currently the second leading cause of death and third main cause of disability throughout the world ${ }^{2}$. Among all risk factors, cardiac embolism is the cause of stroke in patients with age $<40$ years while in the individuals aged 41-50 years, atherosclerosis is reported to be the main cause leading towards the debilitating condition ${ }^{3}$. Around $75 \%$ of stroke survivors are suffered from the disability of upper limb with limited functional activities and upper extremity dysfunction ${ }^{4}$ whereas distal parts of upper extremity such as hand and forearm are mostly affected by stroke. Number of studies reported that approximately $70 \%$ limitation in fine motor activities of hand has shown after one week of stroke while $41 \%$ limitation has observed within third month post-stroke ${ }^{5}$. Moreover, the patient is unable to perform daily living activities due to upper extremity impairment. These basic activities are the survival living skills such as dressing, bathing, and toileting, transferring or feeding. Despite the adverse consequences of stroke, its rehabilitation is primary objective to improve the functional abilities of patients to minimize activity limitation and participation restriction. It has been observed that patients with post-stroke upper extremityimpairments laidheavy burden on their families and caregivers ${ }^{6}$. Furthermore, $>50 \%$ of patients with upper limb dysfunction remain frustrated with increased anxiety levels ${ }^{7}$ due to motor impairments of the upper extremity leading patient towards the poor perception regarding health related quality of life $(\mathrm{HRQOL})^{8}$. However, depression in stroke patient is common because of slow recovery as $33 \%$ of stroke survivor showed the symptoms of depression at the acute stage while $34 \%$ showed symptoms at the later stage $^{9}$ therefore, the core objective of stroke rehabilitation is to improve upper limb function in order to make patient independent in activities of daily living (ADLs). According to International Classification of functioning the activity involves participation which will ultimately increases social involvement and improves overall health status. Wakkel and colleagues concluded that $12 \%$ patients regained upper limb function at the sixth month of rehabilitation ${ }^{10}$ whereas quite a few patients experienced difficulty in fingers and hand coordination". Nevertheless, with the passage of time impairment in the motor activity of hand increases which results in the formation of deformity such as contractures, nerve compression therefore rehabilitation after stroke is very essential in order to prevent the development of deformity in the hand. Besides, occupational therapy is the core element of rehabilitation to improve hand function of the patient ${ }^{12}$. Since upper extremity is involve in almost all functional activity, though a patient is trained for one hand skills too but bilateral involvement is required in many tasks. Secondly the disuse of affected arm will lead to not only disability and deformity but disfigurement and poor participation. Finally the health and wellbeing of an individual get affected.

Subsequently due to reduced cortical representation in parietal cortex, sensory input to the brain is reduced which particularly affect hand functioning ${ }^{13}$. In order to improve sensory function in stroke patient application of somatosensory stimulation is common ${ }^{14}$. It helps in maintaining the cortical representation of the hand as well as it facilitates movement by motor system ${ }^{15}$. A systemic review revealed that somatosensory stimulation could provide effective outcome in improving motor function ${ }^{16}$. Many rehabilitative approaches are available for improving motor function of upper extremity such as muscle strengthening exercises, transcutaneous electrical stimulation (TENS), constraint induced movement therapy (CIMT), proprioceptive neuromuscular facilitation techniques etc. Though, a repetitive hand and finger exercise with varying loads is a new approach in stroke rehabilitation ${ }^{17}$. Therefore, occupational therapists mustdesign treatment plan according to condition of the patient and the stage of the stroke to improve disabilities and impairments of upper extremity.

In rehabilitation, TENS is an effective device for electrical somatosensory stimulation operated by sending stimulating impulses across the skin surface and nerve stands to the brain which improves motor performance and corticospinal excitability ${ }^{18}$. TENS is effective, feasible and easily applicable modality for the rehabilitation of stroke patient. According to Dos Santos-Fontes and colleagues, the application of somatosensory electrical stimulation (SES) through TENS could be an effective therapy for some stroke patients whereas $31-36 \%$ improvement as compared to baseline hand function was detected in the mean Action reach arm test ARAT score in stroke patients ${ }^{19}$. Moreover, the application of low-frequency TENS on upper limb showed improvements in motor kinematics and function due to changes in ipsilesional cortical oscillations ${ }^{20}$ thereby induces cortical plasticity in the damaged region of the brain and enhances motor-function related activation ${ }^{21}$. Number of studies demonstrated that TENS also decreases resting-state low-frequency cortical oscillations which results in spontaneous recovery of hand function of the stroke patients ${ }^{22-23}$. Several studies showed that task related training with strengthening exercises and TENS is effective in decreasing spasticity and functional mobility of upper limb ${ }^{24}$. Furthermore, it has been observed the effects of electrical stimulation assisted task specific upper extremity and hand exercisesresults in the improvement of the motor activity of hand along with the sensory improvement ${ }^{25-26}$. Another study compared the effects of electrical stimulation on the elbow and wrist extensor for twelve weeks and they found the significant improvement in hand and wrist motor activity ${ }^{27}$ where it has been publicized that sufficient 
electrical stimulation is required for motor cortex's modulation of reorganization during performance motor activities which could provide beneficial results for improving motor function, especially in stroke rehabilitation ${ }^{28}$. Thus, this study aims to determine the benefits of TENS along with conventional occupational therapy in the rehabilitation of stroke patients to ascertain the optimal recovery of hand function. Apart from Activities of daily living even Instrumental activities that need motor involvement of upper extremity also improve.

\section{METHODOLOGY}

\section{Study Setting}

The study was conducted at Occupational Therapy department of outpatient or day care at two of the tertiary care hospitals in Karachi.

\section{Study Design}

It is a quasi-experimental study, comparing the conventional occupational therapy effect to the combination of TENS with Conventional therapy at Stroke patients.

\section{Target Population}

Patients with acute phase or one year post-stroke aged 20-80 years were enrolled.

\section{Duration Of Study}

Six months.

\section{Sampling Technique}

Probability Sampling Technique-Simple random sampling was used.

\section{Sample Size}

A sample size of 76 was calculated using $\mathrm{WHO}$ software.

\section{Inclusion Criteria}

- Stroke survivors with acute phase to one year post-stroke aged 20-80 years old were included.

\section{Exclusion Criteria}

Patients with severe cognitive impairment such as memory loss, aphasic and uncontrolled medical conditions were excluded.

\section{Data Collection Procedure}

A sample of 76 participants were randomly divided into experimental and control groups. Both the groups received intervention for 5 days/week for the duration of 45 minutes for total 3 weeks. The protocols of intervention are described as follows:

\section{- Treatment Protocol For Group-A}

Group-A $(n=38)$ received Low Frequency Transcutaneous Electrical Nerve Stimulation (LF-TENS) on the extensors of elbow and wrist with frequency set at $3 \mathrm{~Hz}$ and pulse duration of $400 \mu$ sfor the duration of 20 minutes along with conventional therapy comprised of passive ROM, upper extremity stretchings and manual dexterity exercises through physio-ball and coin fine activities for 25 minutes.

\section{- Treatment Protocol For Group-B}

Group B ( $n=38)$ received conventional occupational therapy comprised of purposeful and functional therapeutic activities along with passive ROM, upper limb stretching, weight bearing, Bo bath technique and manual dexterity for 45 minutes.

\section{Data Collection Tool}

Data was collected at baselinepreliminary to the intervention and after the end of treatment on two outcome measures:

- Fugal- Meyer Assessment of Upper Extremity (FMA-UE) is a comprehensive, valid and reliable evaluation tool to assess post-stroke sensorimotor function. FMA-UE is a 3-point ordinal scale comprises of 33 items in which motor domain is widely used due to its primary value in monitoring functional recovery.

- Action reach arm test (ARAT) isa standardized tool to assess upper extremity activity capacity. ARAT is a 4-point ordinal scale comprises of 19 items to evaluate post-stroke coordination, dexterity and functional recovery.

\section{Data Analysis}

Data was entered and analyzed on SPSS (Statistical Package for Social Sciences) version 20. Demographic characteristics of the participants were determined through frequency, mean and standard deviations. While based on symmetrical normality assumption, paired t-test was applied for within the group analysis and independent t-test was run for the comparison of post mean values between the experimental and control groups whereas $p<0.05$ was considered significant.

\section{Ethical Considerations}

Participant's information was ensured to be kept confidential. Prior to the intervention, informed consent was taken by each participant that highlighted the possible risks, benefits, confidentiality and rights of withdrawal from the study at any time.

\section{RESULTS}

Total 76 participantswith acute or one year post-stroke history recruited in the study, 38 in each group respectively with mean age of $76.5 \pm 9.7$ in Group A have19 male and 18 female subjects correspondingly whereas in Group B, 20 participants were male and 18 were females with mean age of77.3 \pm 9.3 . Participants were assessed at baseline and post 3-weeks intervention on FMA-UE and ARAT; moreover no loss of follow-upwas reported. Demographic characteristics of participants are shown in Table-1. 


\begin{tabular}{|l|c|c|}
\hline \multicolumn{3}{|c|}{ Table 1. Demographic Characteristics } \\
\hline & $\begin{array}{c}\text { Group -A } \\
(\mathrm{n}=\mathbf{3 8})\end{array}$ & $\begin{array}{c}\text { Group -B } \\
(\mathrm{n}=\mathbf{3 8})\end{array}$ \\
\hline Age (years) & $76.5 \pm 9.7$ & $77.3 \pm 9.3$ \\
\hline Gender & $\begin{array}{c}19 \text { Males, 19 } \\
\text { Females }\end{array}$ & $\begin{array}{c}20 \text { Males, 18 } \\
\text { Females }\end{array}$ \\
\hline \multicolumn{3}{|c|}{ Stroke type } \\
\hline Left Sided & $18.0 \pm 6.7$ & $18.0 \pm 6.2$ \\
\hline Hemorrhagic & $5 \pm 1.9$ & $5 \pm 1.7$ \\
\hline Ischemic & $4.8 \pm 1.2$ & $3.6 \pm 1.7$ \\
\hline $\begin{array}{l}\text { Stroke } \\
\text { Duration }\end{array}$ & $13.1(8.5-25.6)$ & $33.3(26.0-39.0)$ \\
\hline
\end{tabular}

In within the group analysis, both the groups showed improvement in sensorimotor function on FMA-UE where Group-A scores significantly improved from $74.89 \pm 9.35$ to $93.3 \pm 12.62$ while Group-B showed improved scores as well from $68.87 \pm 14.63$ to 77.89 \pm 13.96 post 3 -weeks intervention $(p<0.05)$ respectively. Similarly on ARAT, Group-A scores significantly improved from was $20.42 \pm 9.98$ to $27.66 \pm 10.13$ whereas Group-B showed moderate improvement in mean scores of hand function from $18.63 \pm 8.17$ to $25.13 \pm 8.05$

$(p<0.05)$ post intervention as shown in Table2, Figure1.

Therefore, it is ascertain that TENS along with conventional therapy is effective in optimal recovery of hand function among stroke patients as illustrated in Table 3, Figure 2.

\begin{tabular}{|c|c|c|c|c|c|c|}
\hline & & $\begin{array}{c}\text { FMA - UE } \\
\text { Mean } \pm \text { S.D. }\end{array}$ & $\begin{array}{c}\text { Mean } \\
\text { Difference }\end{array}$ & $\begin{array}{c}\text { ARAT } \\
\text { Mean } \pm \text { S.D. }\end{array}$ & $\begin{array}{c}\text { Mean } \\
\text { Difference }\end{array}$ & $P$-value \\
\hline \multirow{2}{*}{ Group - A } & Pre & $74.89 \pm 9.35$ & \multirow{2}{*}{18.41} & $20.42 \pm 9.98$ & \multirow{2}{*}{7.24} & \multirow{2}{*}{$<0.05$} \\
\hline & Post & $93.3 \pm 12.62$ & & $27.66 \pm 10.13$ & & \\
\hline \multirow{2}{*}{ Group - B } & Pre & $68.87 \pm 14.63$ & \multirow{2}{*}{9.02} & $18.63 \pm 8.17$ & \multirow{2}{*}{6.5} & \multirow{2}{*}{$<0.05$} \\
\hline & Post & $77.89 \pm 13.96$ & & $25.13 \pm 8.05$ & & \\
\hline
\end{tabular}

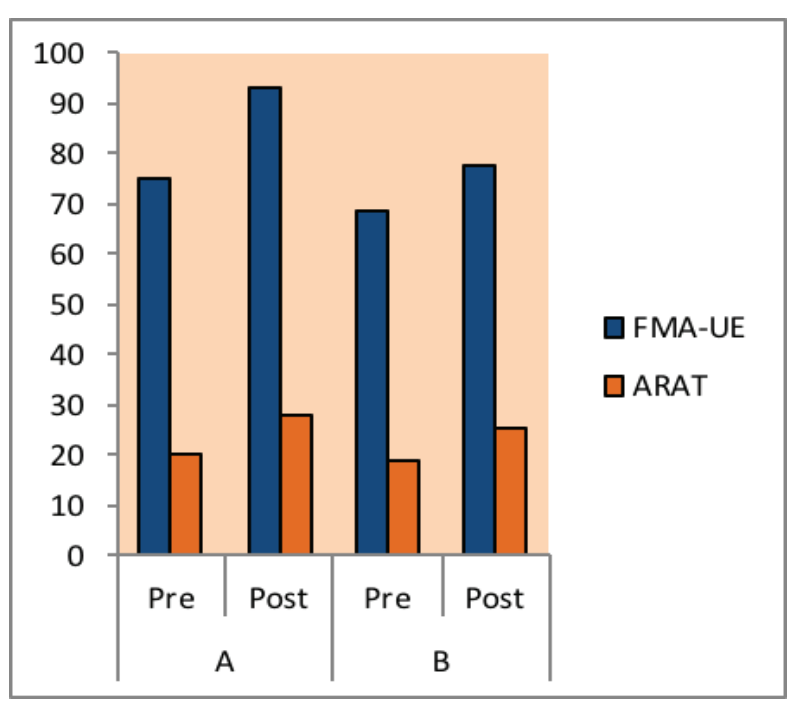

Figure-1 shows pre-post mean differences on FMA-UE and ARAT

The comparison of post-intervention mean values on FMA and ARAT outcome measures revealed that Group-A mean scores significantly improved sensorimotor and activity capacity of hand function in post-stroke patients in comparison to Group-B which also showed moderate significant results. Moreover, strong positive correlation was determined between muscle activation and improvement of motor functioning of hand in patients who were treated with TENS.

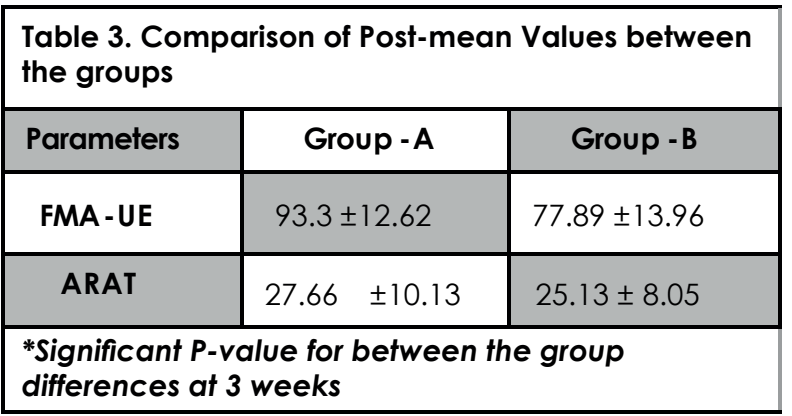

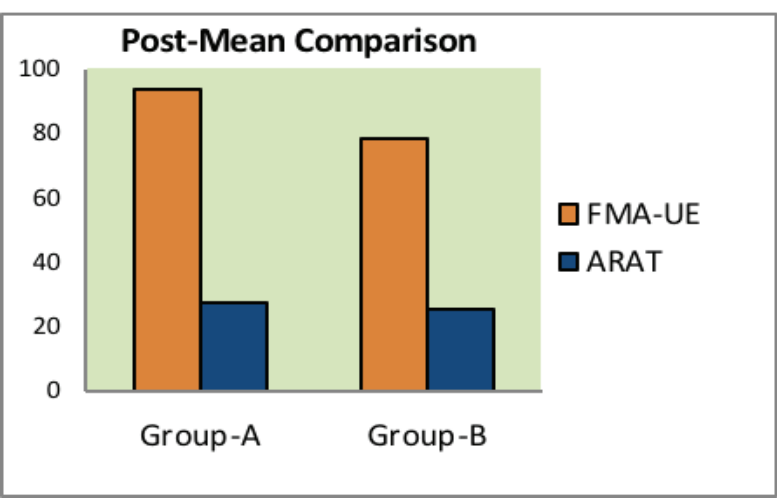

Figure 2. Post-Mean Comparison of Group A and B 


\section{DISCUSSION}

The results of this studyshowed that the application of somato-sensory stimulation through TENS along with conventional manual therapy significantly improved the motor function of the hand. Therefore, it has been established that somatosensory electrical stimulation through TENS is the core part of stroke rehabilitation along with manual exercises. In contrast, Grant et al revealed that somatosensory stimulation through TENS was not effective in recovery of motor function of hand and motor system for movements in stroke patient ${ }^{29}$. According to the study of Pollock and colleagues repetitive manual exercises did not proficiently recover the functions of hand and fingers in the patients with acquired brain injury ${ }^{8}$. However, our study showed better outcomes with respect to the motor function of hand by applying somatosensory stimulation through TENS along with conventional Occupational therapy. On the other hand, findings of our study are consistent with the results of Laufer and Elboim-Gabyzonthat showed sensory stimulation with TENS provide better outcome by leading the long lasting cortical plasticity ${ }^{30}$. Moreover, Hatem and colleagues revealed that combination therapy of high-frequency TENS and rehabilitation treatment provide better results as compared to the rehabilitation treatment alone for stroke patients with upper extremity disabilities and impairments and theyascertained that low-frequency TENS has no effect on improving disabilities of upper extremity ${ }^{31}$. Whereas, our study provided a strong evidence that application of low frequency TENS leads to the improvement of motor function of hand and its kinematics due to change in the ipsilesional cortical oscillations ${ }^{14}$. Furthermore, Sonde et al showed that the application of low frequency TENS for 60 minutes results in increase of motor function of hand while our study demonstrated an effective application of TENS for the duration of 20 minutes to enhance motor function. Furthermore, Tu-Chan and colleagues evaluated the effects of repetitive application of somatosensory electrical stimulation through TENS that showed significant improvements in hand-finger movements on ARAT ${ }^{6}$, the findings are consistent with our study outcomes where both experimental and control group showed significant improvementson FMA-UE and ARAT with respect to treatment time. Besides, Kusoffsky, Wadell and Nilsson revealed that Low-frequency TENS optimizes motor control mechanism; however, it doesn't improve the sensory function of stroke-affected arm and hand ${ }^{32}$ whereas afferent stimulation with the help of low-frequency TENS uses plasticity and capacity of the brain for reorganization. In general, Low-frequency TENS therapy provides successful results in both early and later phase of stroke recovery.

\section{CONCLUSION}

It was concluded that conventional occupational therapy along with TENS significantly improved the hand function in acute stroke patients as compared to limiting to conservative occupational therapy. Thuscombination of TENS with occupational therapy enhances motor activity of impaired muscles that leads to optimal functional recovery of stroke survivors.

\section{REFERENCES}

[[1] Vijayan M, Reddy PH. Stroke, vascular dementia, and Alzheimer's disease: molecular links.J Alzheimers Dis. $2016 ; 54(2): 427-43$

[2] Power A, Chan K, Singh SK, Taube D, Duncan N. Appraising stroke risk in maintenance hemodialysis patients: a large single-center cohort study. American journal of kidney diseases. 2012 ;59(2):249-57.

[3] Tanaka H, Toyonaga T, Hashimoto H. Functional and occupational characteristics predictive of a return to work within 18 months after stroke in Japan: implications for rehabilitation. International archives of occupational and environmental health. 2014 ;87(4):445-53.

[4] Winters C, van Wegen EE, Daffertshofer A, Kwakkel G. Generalizability of the proportional recovery model for the upper extremity after an ischemic stroke. Neurorehabilitation and neural repair. 2015 Aug;29(7):614-22.

[5] Connell LA, Tyson SF. Measures of sensation in neurological conditions: a systematic review. Clinical rehabilitation. 2012 Jan;26(1):68-80.

[6] Corbetta D, Sirtori V, Castellini G, Moja L, Gatti R. Constraint-induced movement therapy for upper extremities in people with stroke. Cochrane Database of Systematic Reviews. 2015(10).

[7] Beleckas CM, Wright M, Prather $\mathrm{H}$, Chamberlain A, Guattery J, Calfee RP. Relative prevalence of anxiety and depression in patients with upper extremity conditions. The Journal of hand surgery. 2018;43(6):571-e1

[8] Lincoln NB, Brinkmann N, Cunningham S, Dejaeger E, De Weerdt W, Jenni W, Mahdzir A, Putman K, Schupp W, Schuback B, De Wit L. Anxiety and depression after stroke: a 5 year follow-up.Disabil Rehabi. 2013;35(2):140-5.

[9] Hackett ML, Pickles K. Part I: frequency of depression after stroke: an updated systematic review and meta-analysis of observational studies. International Journal of Stroke. 2014;9(8):1017-25.

[10] Coupar F, Pollock A, Rowe P, Weir C, Langhorne $P$. Predictors of upper limb recovery after stroke: a systematic review and meta-analysis. Clinical rehabilitation. 2012 Apr;26(4):291-313.

[11] Ranganathan R. Reorganization of finger coordination patterns through motor exploration in individuals after stroke. Journal of neuroengineering and rehabilitation. 2017;14(1):90.

[12] Pollock A, Farmer SE, Brady MC. Interventions to improve arm and hand function in people after stroke. The Cochrane Library. 2014;(11).

[13] Borich MR, Brodie SM, Gray WA, lonta S, Boyd LA. Understanding the role of the primary somatosensory 
cortex: Opportunities for rehabilitation. Neuropsychologia. 2015;79:246-55.

[14] Bolognini N, Russo C, Edwards DJ. The sensory side of post-stroke motor rehabilitation. Restorative neurology and neuroscience. 2016 Jan 1;34(4):571-86.

[15] Chen X, Liu F, Yan Z, Cheng S, Liu X, Li H, Li Z. Therapeutic effects of sensory input training on motor function rehabilitation after stroke. Medicine. 2018;97(48).

[16] Connell LA, Tyson SF. Measures of sensation in neurological conditions: a systematic review. Clinical rehabilitation. 2012;26(1):68-80.

[17] Hatem SM, Saussez $G$, della Faille $M$, Prist $V$, Zhang $X$, Dispa D, Bleyenheuft $Y$. Rehabilitation of motor function after stroke: a multiple systematic review focused on techniques to stimulate upper extremity recovery. Frontiers in human neuroscience. 2016;10:442..

[18] Rollnik JD. May clinical neurophysiology help to predict the recovery of neurological early rehabilitation patients?.BMC Neurol. 2015;15(1):239.

[19] Dos Santos-Fontes RL, Ferreiro de Andrade KN, Sterr A, Conforto AB. Home-based nerve stimulation to enhance effects of motor training in patients in the chronic phase after stroke: a proof-of-principle study. Neurorehabil Neural Repair. 2013;27(6):483-90.

[20] Finnigan S, Wong A, Read S. Defining abnormal slow EEG activity in acute ischaemic stroke: Delta/alpha ratio as an optimal QEEG index Clin Neuropsycol 2016;127(2):1452-9.

[21] Rollnik JD. May clinical neurophysiology help to predict the recovery of neurological early rehabilitation patients?BMC Neurol. 2015;15(1):239.

[22] Tu-Chan AP, Natraj N, Godlove J, Abrams G, Ganguly K. Effects of somatosensory electrical stimulation on motor function and cortical oscillations. J Neuroeng Rehabil. 2017;14(1):113.

[23] Veldman MP, Maffiuletti NA, Hallett M, Zijdewind I. Hortobágyi T. Direct and crossed effects of somatosensory stimulation on neuronal excitability and motor performance in humans. Neuroscience \& Biobehavioral Reviews. 2014;47:22-35.

[24] Kim TH, In TS, Cho HY. Task-related training combined with transcutaneous electrical nerve stimulation promotes upper limb functions in patients with chronic stroke. Tohoku J Exp Med. 2013;231 (2):93-100.

[25] Sullivan JE, Hurley D, Hedman LD. Afferent stimulation provided by glove electrode during task-specific arm exercise following stroke. Clinical rehabilitation. 2012 Nov;26(11):1010-20.

[26] Pan LL, Yang WW, Kao CL, Tsai MW, Wei SH, Fregni F, Chen VC, Chou LW. Effects of 8-week sensory electrical stimulation combined with motor training on EEG-EMG coherence and motor function in individuals with stroke. Scientific reports. 2018 Jun 15:8(1):1-0.

[27] Okuyama K, Ogura M, Kawakami M, Tsujimoto K, Okada K, Miwa K, Takahashi Y, Abe K, Tanabe S, Yamaguchi T, Liu M. Effect of the combination of motor imagery and electrical stimulation on upper extremity motor function in patients with chronic stroke: preliminary results. Therapeutic advances in neurological disorders. $2018 ; 11: 1756286418804785$. .

[28] Cho HY, In TS, Cho KH, Song CH. A single trial of transcutaneous electrical nerve stimulation (TENS) improves spasticity and balance in patients with chronic stroke. The Tohoku journal of experimental medicine. 2013;229(3):187-93.

[29] Grant VM, Gibson A, Shields N. Somatosensory stimulation to improve hand and upper limb function after stroke-a systematic review with meta-analyses. Top Stroke Rehabil.2018;25(2):150-60. [30] Laufer Y, Elboim-Gabyzon M. Does sensory transcutaneous electrical stimulation enhance motor recovery following a stroke? A systematic review.Neurorehabil Neural Repair. 2011 ;25(9):799-809

[31] Hatem SM, Saussez $G$, della Faille $M$, Prist $V$, Zhang $X$, Dispa D, Bleyenheuft $Y$. Rehabilitation of motor function after stroke: a multiple systematic review focused on techniques to stimulate upper extremity recovery.Front Hum Neurosci. 2016;10:442 [32] Scalha TB, Miyasaki E, Lima NM, Borges G. Correlations between motor and sensory functions in upper limb chronic hemiparetics after stroke. Arquivos de neuro-psiquiatria. 2011 ;69(4):624-9. 